\title{
First results of the Maastricht brace in the treatment of adolescent idiopathic scoliosis
}

\author{
Dirk Schrander ${ }^{*}$, Joris Hermus, Helma Voets, Mark van den Boogaart, Paul Willems, Lodewijk van Rhijn \\ From 11th International Conference on Conservative Management of Spinal Deformities - SOSORT 2014 \\ Annual Meeting \\ Wiesbaden, Germany. 8-10 May 2014
}

\section{Background}

The Maastricht brace (M-brace) was developed to improve compliance and associated efficacy of brace treatment in adolescent idiopathic scoliosis (AIS). Initial pressure measurements in the M-brace revealed a higher corrective pressure as compared to the Boston brace, and a better patient reported quality of life, as measured with the SRS 22 and Brace questionnaire. We present the first results of the efficacy in terms of curve correction of the M-brace in AIS.

\section{Aim}

The aim of this study was to evaluate the in-brace curve correction of the Maastricht brace and to determine the effect of increased wearing comfort on treatment efficacy.

\section{Design}

Retrospective cohort study.

\section{Methods}

A total of 46 patients (mean age of 13 years) with mild to moderate AIS, who have been treated with the Mbrace since January 2011, were included. The correction effectiveness of the brace was evaluated by comparing the primary and secondary curves on bending $x$-rays with those on standard postero-anterior full spine radiographs with and without M-brace. The degree of correction in the $\mathrm{M}$ - brace was then expressed as a percentage of the correction as achieved in the bending radiographs. As a control group four patients were also fitted a Boston brace, in order to compare the in-brace correction between the braces.

\section{Results}

There were 38 patients with a primary thoracic curve, and 8 patients with a primary lumbar curve. The average primary curve angle measured in Cobb degrees was $34.7^{\circ} \pm 11.3^{\circ}$. The average primary curve angle in bending $x$-rays was $15.5^{\circ} \pm 8.3^{\circ}$. In the M-brace the primary curve was $25.4^{\circ} \pm 10.1^{\circ}(\mathrm{p}<0.01)$. This is an in-brace correction of $48 \%$. The control group had an in-brace correction of $49.7 \%$ in the Boston brace versus $45.1 \%$ in the M-brace $(\mathrm{p}=0.21)$.

\section{Conclusions}

These preliminary results demonstrate an adequate inbrace correction of the M-brace, which is comparable to corrections found in current literature and similar to the in-brace correction of the Boston brace in the control group. Given the relationship between compliance and wearing comfort, the $\mathrm{M}$-brace is, without compromising treatment efficacy, a promising new brace treatment for adolescent idiopathic scoliosis.

Published: 4 December 2014

doi:10.1186/1748-7161-9-S1-034

Cite this article as: Schrander et al:: First results of the Maastricht brace

in the treatment of adolescent idiopathic scoliosis. Scoliosis 2014 9(Suppl 1): 034 . 